\title{
A Resilience Enhancement Model for Complex Distribution Network Coupling with Human Resources and Traffic Network
}

\author{
Biyun Chen, Yumo Shi $\mathbb{D}$, and Yanni Chen \\ Guangxi Key Laboratory of Power System Optimization and Energy Technology, Guangxi University, Nanning 530004, China \\ Correspondence should be addressed to Yumo Shi; 1812301028@st.gxu.edu.cn
}

Received 2 May 2021; Accepted 15 May 2021; Published 7 June 2021

Academic Editor: Chun Wei

Copyright ( $\odot 2021$ Biyun Chen et al. This is an open access article distributed under the Creative Commons Attribution License, which permits unrestricted use, distribution, and reproduction in any medium, provided the original work is properly cited.

\begin{abstract}
Resilience is the ability of a system to withstand and recover from deliberate accidents; as to distribution systems, it means the ability to withstand and recover from natural disasters or other serious accidents and ensure electricity supply. Generally, promotion strategies of distribution network resilience mostly focus on electrical topology planning and reinforcement. The operation strategies in emergency repair stage are frequently ignored, especially the complex coupling relationship between distribution network, traffic network, and maintenance teams. A model of resilience improvement for a complicated distributiontraffic-human coupling system under hurricane disasters considering is presented. Firstly, based on the influence spreading mechanism of a hurricane acting on the distribution and traffic part of the system, a fault analysis model of rush repair is constructed. Secondly, according to the function of human resources in shortening the repair time and improving system resilience, an optimization model of emergency repair strategy is proposed. Taking into account the repair demand, traffic cost, and personnel operating and executing ability, the optimized strategy can minimize the social loss in the whole repair and recovery process after hurricane disaster. Furthermore, three indices, including system adaptability, repair rate, and economic loss rate, are proposed to quantify the resilience of distribution network. Finally, case studies on the IEEE33 bus system are implemented to verify the effectiveness of the proposed model.
\end{abstract}

\section{Introduction}

Extreme weather events, in particular hurricanes, have a great impact on electric infrastructures, resulting in extensive damages and significant economic and social losses. As a vulnerable part of the electricity infrastructure, distribution network suffers most outages which caused considerable inconvenience for residents living in disaster areas [1]. Therefore, enhancing the distribution network resilience becomes a key task for protecting power systems from extreme weather events.

Resilience, first proposed by Ecologist Holling, describes the ability of species to absorb disturbances and survive. In the context of power system, resilience reflects "the ability of power systems to withstand low-probability high-impact incidents in an efficient manner while ensuring the least possible interruption in supply of electricity, and further enabling a quick recovery and restoration to the normal operation state" [2]. There are various studies of distribution network resilience. In [3], a resilience assessment matrix was put forward to evaluate how the system is suffering and recovering from extreme disturbances, especially natural disasters, from technical, organizational, social, and economic perspectives. Enhancing the resilience of distribution grids from a technical way is the current mainstream approach. A resilience-oriented design (ROD) model was built to obtain the optimal hardening strategy, represented as a two-stage stochastic mixed-integer problem [4]. The first stage was to make the ROD decisions, like hardening lines and installing distributed generations (DGs). Then the second stage evaluated the system operation cost and repair cost. In [5], an optimization configuration model was proposed to improve the disaster resistance of distribution network, considering the impact of automatic switch in addition. The configuration scheme obtained has preferable economic benefits and resilience improvement efficiency. A 
two-stage stochastic programming approach was presented to optimize the scheduling of a resilient microgrid (MG), considering the impact of natural disasters and the prevailing uncertainties associated with wind energy, electric vehicles, and real-time market prices [6, 7]. A comprehensive summary of distribution network resilience was presented in $[8,9]$, including evaluation models such as resilience triangle model and trapezoid model, and introducing several methods to define resilience indicators, such as area definition method, probability definition method, cost definition method, and weighted definition method. Among them, the definition method of power loss area is the most widely used.

With the modernization of cities, some researchers have noticed that the coupling between the important infrastructure systems will affect the distribution network resilience. Considering the interdependence of the urban transportation system (UTS) and power distribution system (PDS), a trilevel optimization model was proposed to improve the coupled system resilience by line hardening and DGs placement [10]. The high penetration of electric vehicles (EVs) further complicates the coupling between the UTS and PDS [11]. The electricity demand uncertainty at each charging/swapping facility depends on the traffic demand of EVs, resulting in the PDS load perturbation and affecting the distribution of traffic flow in turn. A robust dispatch method was proposed to maintain the feasibility of alternating current (AC) power flow constraints considering the impact of road congestion on route choices of VEs [12]. In [13], a two-stage robust optimization model was proposed for expansion planning of coupled active distribution and transportation based on the interaction of roads, distribution lines, DGs, energy storage systems, and charging facilities. A quantitative resilience assessment framework is proposed for the traffic-distribution network, considering both the spatial and temporal impacts of hurricanes [14]. In [15], a resilience enhancement strategy was proposed to improve the load survivability of distribution network under extreme weather events, taking into consideration the interaction between the information system and distribution system. To improve the resilience of distribution network cyber physical system (distribution network CPS) under the network attacks, authors in [16] proposed a method to analyze and evaluate the system risk based on the correlation matrix model of the coupled system.

However, most of the studies improve the distribution network resilience by grid hardening strategy, while paying insufficient attention to the operation measures in postdisaster recovery process. In the postdisaster recovery process, appropriate repair-recovery strategies can shorten the emergency repair time and improve the power supply capacity of critical loads, which significantly enhances the distribution network resilience. Social factors play an important role in the execution of repair-recovery strategies. In [17], a management strategy, considering the impact of social factors, was proposed to reduce postdisaster repair costs and improve comprehensive benefits. A framework was proposed to analyze the human factors and rank error factors for improving system operation reliability [18]. In
[19], an emergency repair model was proposed to decrease the repair cost considering the specialty of the repair team and the needs of the faulting task, quantifying the influence of human resources by the utility theory. Soon after, the emergency repair model was strengthened to deal with the multifault in distribution network, considering the cooperation between the repair teams [20]. Moreover, a repairrecovery dynamic model was established to solve the multifault in active distribution network with more layers and scenes based on a multiagent system [21]. These studies focused on the repair-recovery strategy itself and failed to associate repair-recovery strategy with system resilience. In [22], the authors proposed a model to evaluate the distribution network resilience, considering the impact of grid reconstruction and repair-recovery strategy. Unfortunately, the model ignored the impact of human resources in the emergency repair process and simplified the repair process which does not conform to reality.

In this paper, studies focused on the resilience during the emergency repair process after extreme weather events (e.g., hurricane disasters) hit distribution network. The emergency repair environment of distribution network is a complicated distribution-traffic-human coupling system, in which both the distribution and traffic part of the coupling system suffered from the extreme weather events and influenced the repair effect by their physical interdependence. The changes in road conditions under extreme weather will influence the route choices of maintenance staff and increase the transportation cost during the emergency repair stage, which causes the operating cost of the distribution network to increase linearly [13]. Meanwhile, the changes in traffic time will directly affect the repair time which is significant to the system resilience. It is necessary to consider the coupling impact of the distribution-traffic network during the emergency repair stage. Besides, the impact of human resources in emergency repair process also affects the repair effect. For example, repair personnel with different capabilities have significantly different time-consuming and repair results when performing the same repair task. Figuring out and applying the mechanism of human resources in the complicated distribution-traffic coupling system during the emergency repair process could enhance the system resilience.

On these basics, a resilience enhancement strategy is proposed for the complex distribution network coupling with human resources and traffic network against hurricane disasters. First, analyze the characteristics of hurricane disasters and figure out the physical vulnerability of distribution-traffic coupling network related to wind speed. Thus, a fault analysis model is established to generate random failure scenes considering the wind speed, equipment failure probability, and traffic flow speed. Second, according to the postdisaster emergency repair process, determine the mechanism of human resources in improving the complicated distribution-traffic network resilience. An optimization model of emergency repair strategy is proposed, considering repair demands, traffic cost, and the operation ability of the repair team, to minimize the social loss caused by the whole emergency repair process. The obtained repair 
strategy has good execution and rapidity which is crucial to resilience. Finally, three indexes, including the adaptive rate of the system, repair rate, and economic loss rate, are proposed for quantitative evaluation on the complex distribution network resilience.

The rest of the paper is organized as follows. Section 2 discusses the process of generating random scenes considering the impact of the hurricane on the complicated distribution-traffic coupling system. Section 3 proposes the mathematical formulation of the emergency repair model considering the human resources. Section 4 presents the general framework and indexes for enhancing and evaluating the complex distribution network resilience. Numerical case studies are presented in Section 5 to demonstrate the effectiveness of the proposed method. Finally, Section 6 concludes the study.

\section{Random Scene Model}

2.1. Component Failure Model Based on Dynamic Wind Field. Wind speed plays a major role in power system planning, which would determine the design strength of components [23]. In this case, the failure probability of a structure or structural component is closely relevant to the maximum wind speed that it suffers during the hurricane. Thus, in order to obtain the wind speed at a certain point within the disaster area during the entire hurricane and calculate their failure probability, a dynamic wind field model should be established for simulation.

2.1.1. Dynamic Wind Field Model. Assume that the influence range of the hurricane can be approximated as a circle, and the hurricane has no detrimental influence upon the distribution network outside its outer boundary. And within the region that hurricane affected, the wind speed $v$ at each point can be given as a function of the distance $d$ to the eye, i.e., the hurricane center. When $d$ increases from 0 to the maximum wind speed radius, wind speed is monotone increasing; when $d$ increases from the maximum wind speed radius to hurricane coverage radius, wind speed is monotone decreasing [24]. This functional relationship is shown in the following equation:

$$
v(d)= \begin{cases}K v_{m}\left(1-\exp \left[-\frac{d}{r_{m v}} \ln \left(\frac{K}{K-1}\right)\right]\right), & 0 \leq d<r_{m v}, \\ v_{m} \exp \left[-\left(\frac{\ln \beta}{r_{s}-r_{m v}}\right)\left(d-r_{m v}\right)\right], & r_{m v} \leq d \leq r_{s}, \\ 0, & d>r_{s},\end{cases}
$$

where $K$ is an empirical parameter, $v_{\mathrm{m}}$ is the maximum sustained wind speed at time $t, r_{\mathrm{mv}}$ is the radius to maximum wind speed, $r_{s}$ is the radius of the area affected by the hurricane, and $\beta$ is the hurricane boundary factor, which represents the ratio of the maximum gradient wind speed to the boundary wind speed.
After the hurricane landed, wind speed would weaken over time by the obstructions on land. Essentially, the wind field is a time-varying field of a concentric circle in translational motion. Capture a certain time section in the process of movement, the static wind field of the hurricane can be modeled as follows:

$$
h=\left\{v_{m}, r_{m v}, r_{s}, K, \beta, \varphi, \psi\right\},
$$

where $\varphi$ are $\psi$ are the latitude and longitude of the eye location, respectively.

Then the hurricane dynamic wind field can be represented by a series of sequentially static wind field along the hurricane path $p_{\mathrm{h}}$ as follows:

$$
\begin{aligned}
H & =\left\{h_{a}, a=0,1, \ldots,|H|\right\}, \\
h_{a} & =\Gamma\left(h_{0} \mid \alpha, p_{h}, v_{h}, \Delta T_{a}\right),
\end{aligned}
$$

where $|\cdot|$ is the number of elements in the set, $h_{0}$ is the wind field where the hurricane lands, and $h_{a}$ is the wind field where hurricane landed at the time sampling point $a . \Gamma(\cdot)$ represents that $h_{l}$ is a time-varying function of $h_{0}$; $\alpha$ is the hurricane land decay factor; $v_{\mathrm{h}}$ is the hurricane translational speed; $\Delta T_{a}$ is the time elapsed from hurricane landfall (in $h_{0}$ ) to the sampling point $a$.

\subsubsection{Component Failure Model of Distribution Network.} The distribution network components mainly include transmission lines, transformers, and switches, including section switches and tie switches. The topological structure of a distribution network could be simplified by making the source-side busbar as the root node, switches and transformers as normal nodes, and transmission lines as edges, separated by switches.

Pole-mounted transformers and switches are vulnerable components when the urban medium-voltage distribution network is invaded and harassed under hurricane scenarios. Furthermore, within some dense vegetation areas, tall vegetation will be toppled over on the line by the gale, causing the collapse or fracture of poles, which leads to the failure of power supply [25]. The outage probability of components in the distribution network is mainly affected by the wind speed of hurricane, and it usually meets the lognormal distribution of wind speed [26]:

$$
p_{i, t}=\Phi\left[\frac{\ln \left(v_{i, t}\right)-\lambda_{i}}{\xi_{i}}\right], \quad \forall i \in \Omega_{L}, t \in \Omega_{T},
$$

where $p_{i, t}$ is the outage probability of equipment $i$ at time $t$, $v_{i, t}$ is the wind speed that equipment $i$ suffers at time $t$, and $\lambda_{i}$ and $\xi_{i}$ are, respectively, the logarithmic mean and the logarithmic standard deviation of intensity of equipment $i$. $\Omega_{T}$ is a hurricane-affected moment set which contains the moment from the first outage of the distribution network to the last one. $\Omega_{L}$ is a controller switching equipment set in disaster areas.

2.2. Traffic Network Model of Emergency Repair Process. Hurricane will not only cause part of the distribution network components to fail but also bring adverse effects 
to the traffic network, which increases the disaster risk of the complicated distribution-traffic coupling system and the complexity of the emergency repair environment. Research shows that it is mainly caused by the secondary disaster brought by the hurricane itself like strong winds, heavy rain, and storm surge [27]. In the urban traffic network, it is mainly reflected in the phenomenon of bending and collapse of trees on both sides of the road, and the low-lying water accumulation caused by the restricted bearing capacity of the drainage system. Therefore, the carrying capacity of the traffic network in the repair process is mainly affected by terrain features and vegetation.

A typical traffic network model consists of traffic intersections, roads, and signal lights, as shown in Figure 1(a). According to characteristics of the emergency repair, the influence of signal lights is ignored to simplify the model. In this case, its topological structure is shown in Figure 1(b); that is, the roundtrip road between two traffic intersections is regarded as a traffic trunk road, traffic intersections are regarded as nodes, and traffic trunk roads are regarded as edges.

Consequently, based on the topology of the traffic network, adjacency matrix $\mathbf{A}$ is set up to store the correlation between nodes [28]. $\mathbf{A}$ is a symmetric matrix of $n$-order, its diagonal elements are 0 , and $n$ is the number of traffic nodes. The meaning of element $a_{i j}$ in $\mathbf{A}$ is as follows:

(1) $a_{i j}=\omega^{T}$ : there is an adjacency relationship between traffic node $i$ and traffic node $j$, and the corresponding edge weight is $\omega^{T}$.

(2) $a_{i j}=\infty$ : there is no adjacency relationship between traffic node $i$ and traffic node $j$, and the corresponding edge weight is infinite.

It is noted that the value $\omega^{T}$ is calculated based on the wind speeds, terrain features, and vegetation, which represents the traffic network vulnerability:

$$
\begin{gathered}
\omega^{T}=\frac{l_{i j}}{\left(1+d_{i j}\right)}, \\
d_{i j}=\frac{e^{h\left(\left.\bar{v}_{i j}\right|_{\max }\right)}}{1+e^{h\left(\left.\bar{v}_{i j}\right|_{\max }\right)},} \\
h\left(\left.\bar{v}_{i j}\right|_{\max }\right)=\sigma_{i j} \frac{\left(\left.\bar{v}_{i j}\right|_{\max }-v_{o}\right)}{v_{o}} k_{i j},
\end{gathered}
$$

where $l_{i j}$ is the edge length connecting traffic nodes $i$ and $j$, $d_{i j}$ is the road traffic difficulty from traffic node $i$ to $j$, and $\left.\bar{v}_{i j}\right|_{\max }$ represents the average of the maximum wind speed that traffic nodes $i$ and $j$ suffer during the hurricane. $\sigma_{i j}$ is the area vegetation density between traffic nodes $i$ and $j ; k_{i j}$ is the area terrain parameters between traffic nodes $i$ and $j ; v_{o}$ is the parameter of wind speed scale which takes the minimum wind speed in wind level 8 in the wind scale, that is $v_{0}=$ $17.2 \mathrm{~m} / \mathrm{s}$ [29].

The feature of traffic node $i$ can be modeled by set $\mathbf{I}$ as

$$
\mathbf{I}=\{i, \varphi, \psi, \zeta\},
$$

where $\varphi$ and $\psi$ are the latitude and longitude of the traffic node $i$, respectively, and $\zeta$ is the parameter set of repair capability.

$$
\begin{aligned}
\zeta & =\left\{\zeta^{\text {staff }}, \zeta^{\text {resource }}\right\}, \\
\zeta^{\text {staff }} & =\left\{M_{a}, a=0,1, \ldots, k\right\}, \\
\zeta^{\text {resource }} & =\left\{N_{a} a=0,1, \ldots, k\right\},
\end{aligned}
$$

where $\zeta^{\text {staff }}$ represents the operation ability of the repair team, $\zeta^{\text {resource }}$ represents the reserve of repair resources, and $k$ is the number of repair teams stationed at traffic node $i$.

And the repair path $R_{o}$ can be described by a set of corresponding nodes:

$$
\Gamma_{R o}=\left\{I_{a}, a=0,1, \ldots,\left|\Gamma_{R o}\right|\right\}, \quad \forall I \in R o .
$$

2.3. Random Scene. According to the posthurricane environment, the random scene model should take serval factors into consideration, such as the wind field model, the distribution-traffic network fragility model, and the systematic load model. The hurricane field model is established to obtain the wind speed and simulate the failure state of the distribution network and traffic network. A random scene $s$ can be described as

$$
s=\left\{t^{\text {land }}, u_{i, t}^{L}, A_{t}, P_{i, t}\right\}, \quad \forall i \in \Omega_{L}, t \in \Omega_{T},
$$

where $t^{\text {land }}$ is the hurricane landfall time, $u_{i, t}^{\mathrm{L}}$ is the failure state of the distribution network, $A_{t}$ is the adjacency matrix of the traffic network, and $P_{i, t}$ is the active power of the distribution network node $i$ at the time $t$.

Random scene $s$ is generated by the following steps:

(1) The hurricane landing time can be predicted from the relevant meteorological system data. Assume that the probability of hurricane landing obeys a uniform distribution during the predicted period. Sample $t^{\text {land }}$ based on its probability density function.

(2) Sample the failure events $z_{i, t}$ in the disaster area $\left(z_{i, t}=1\right.$, the failure event occurrences; $z_{i, t}=0$, no failure event) by the component failure probability $p_{i, t}$, which is calculated according to (1) to (5). $u_{i, t}^{\mathrm{L}}$ is the failure state set of all controller switching equipment in the disaster area.

(3) Establish the initial adjacency matrix $\mathbf{A}$ based on the topology of the traffic network within the disaster area. Calculate $\omega^{T}$ based on (6) to (8) and obtain $A_{t}$.

(4) Calculate $P_{i, t}$ according to (13) and (14).

For the transformer node,

$$
P_{i, t}=\left(1-p_{i, t}^{\text {trans }}\right) P_{i}^{L B}, \quad \forall i \in \Omega_{L}, t \in \Omega_{T} .
$$

For the nontransformer node, if it is previously in a fault state, $P_{i, t}=0$; otherwise, 


$$
P_{i, t}=\tau_{i}(t) P_{i}^{L B}, \quad \forall i \in \Omega_{L}, t \in \Omega_{T},
$$

where $p_{i, t}^{\text {trans }}$ is the failure rate of transformer node $i$ at time $t$ and $\tau_{i}(t)$ is a probabilistic factor, which is used to describe random volatility of load and obeys normal distribution [30]. $P_{i}^{\mathrm{LB}}$ is the base value of active power of node $i$.

\section{Emergency Repair Model considering Human Resources}

The whole emergency repair process of multiple faults in the distribution network caused by extreme natural disasters can be divided into two stages: network reconfiguration stage and load recovery stage. In the network reconfiguration stage, repair personnel isolates outage components and optimizes the network topology as much as possible to restore the power supply of nonfaulty loads from the transfer channel through switch operation. In the load recovery stage, repair personnel gradually repairs the faults in the distribution network to restore all power supply by the maintenance of repairable faulty equipment and the replacement of spare parts for nonrepairable equipment, according to the repair strategy [31].

Human resources, including repair personnel and repair resources, play an essential role in the whole process of distribution network repair, which mainly reflects on the execution effect of repair strategy. The repair ability preference of repair personnel, the resource allocation and geographic location of repair teams, and the demands of fault tasks will affect the repair effect of fault tasks. Different allocation and maintenance sequences of fault tasks will achieve different repair effects and repair times, which have great significance to improve system resilience. Considering the characteristic of human resources to optimizing repair strategies could obtain a good emergency repair execution effect and shorten the repair time, which finally improve the system resilience.

3.1. Path Planning in Fault Repair Process. The paper divides the repair strategy into three parts: the allocation of repair tasks, maintenance sequence, and path planning. In essence, path planning in emergency repair process is to find the shortest path between two points in the traffic network, so as to achieve the shortest driving time of repair personnel. The construction of the urban distribution network is based on the traffic network frame, which forms a geographically overlapping contrast network. Through the latitude and longitude information, the corresponding points of the distribution network fault node can be identified in the traffic network. Thus, path planning of a single fault task of a repair team can be simplified to a single-source shortest path planning with known traffic network topology. Take into consideration the impact of hurricane and mutual influence of the complicated distribution-traffic coupling system during the emergency repair process, adopting the weight of each topology edge of the traffic network obtained from (6) instead of the geographic length of the road to calculate the driving cost. The minimum programming path solution can be calculated by the Dijkstra algorithm, which is a breadthfirst forward search algorithm based on graph theory. Dijkstra algorithm expands to the outer layer with the starting point as the center and constantly looks for the vertex closest to the starting point in each iteration as the starting point of the next iteration until it extends to the endpoint. The set of vertices in the iteration process is the shortest path between two points. Therefore, the minimum driving cost between each node in the repair process would be obtained [28].

3.2. Repair Task Allocation considering Human Resources. In order to realize the numerical evaluation of human resources, this paper adopts utility theory to describe the heterogeneity of fault demand and team repair ability by constructing a utility function $u_{j}(i)$, which represents the matching degree between the repair team $i$ and repair task $j$ [19]:

$$
\begin{aligned}
& u_{j}(i)= \begin{cases}U_{j}\left(C_{i}^{r}, C_{j}^{t}\right), & v_{i j}=1, \\
0, & v_{i j}=0,\end{cases} \\
& U_{j}\left(C_{i}^{r}, C_{j}^{t}\right)=\sum_{p=1}^{k} \omega_{p} \frac{\beta_{i p}}{\alpha_{i p}}, \\
& C_{i}^{r}=\left(\begin{array}{cccc}
\alpha_{i 1} & 0 & \cdots & 0 \\
0 & \alpha_{i 2} & \cdots & 0 \\
\vdots & \vdots & & \vdots \\
0 & 0 & \cdots & \alpha_{i k}
\end{array}\right)\left(\begin{array}{c}
c_{1} \\
c_{2} \\
\vdots \\
c_{k}
\end{array}\right) \text {, } \\
& C_{j}^{t}=\left(\begin{array}{cccc}
\beta_{j 1} & 0 & \cdots & 0 \\
0 & \beta_{j 2} & \cdots & 0 \\
\vdots & \vdots & & \vdots \\
0 & 0 & \cdots & \beta_{j k}
\end{array}\right)\left(\begin{array}{c}
c_{1} \\
c_{2} \\
\vdots \\
c_{k}
\end{array}\right) \text {, }
\end{aligned}
$$

where $C_{i}^{r}$ is the ability vector of repair team $i, C_{j}^{t}$ is the demand vector of repair task $j$, and $v_{i j}$ is a $0-1$ variable which represents the ability to repair faults alone $\left(v_{i j}=1\right.$, the repair team $i$ could execute repair task $j$ alone; $v_{i j}=0$, the repair team $i$ could not execute repair task $j$ alone). $\alpha_{i k}$ is the ability coefficient of the repair team $i$ with ability $c_{k}, \beta_{j k}$ is the demand coefficient of the repair team $i$ with ability $c_{k}$, and $\omega_{p}$ is the weight of ability $c_{p}$ which sums to $1, p=1,2, \ldots, k$. In this paper, the value of weight coefficient is determined by expert experience [32].

The larger the $u_{j}(i)$ value, the better the repair effect. Divide utility value into levels and use the roulette selection method to assign repair tasks; the obtained repair task allocation can ensure meeting the ability preference of each repair team and achieve a good repair effective. 


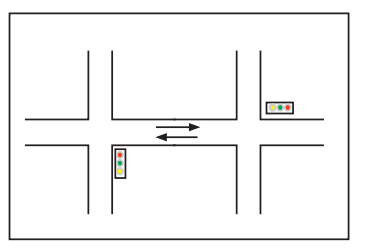

(a)

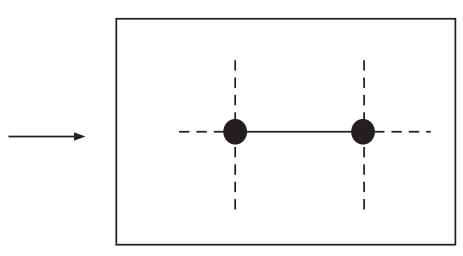

(b)

Figure 1: Traffic network model.

\subsection{Repair Strategy Optimization Model}

3.3.1. Objective Function. When making repair strategies, decision-makers should give priority to restoring the load of great importance and high power. The purpose of emergency repair is to restore the power supply as soon as possible. In this case, whether the operation is economical during the emergency repair stage or not is not of great importance. Thus, repair strategies of the complex distibution network should aim to decrease social loss caused by outages. And previously, the complex coupling relationship between distribution network, traffic network, and repair teams was partly modeled into the optimization model of repair task allocation and path planning, whose results will be called parameters in the following model.

The proposed formulation is introduced as follows, which represents the weighted sum of the total social losses in the whole repair process:

$$
\begin{aligned}
& \min f(X)=\sum_{i=1}^{m+n} t\left(x_{i}\right) \sum_{d j=1}^{3} \omega_{d j}\left(x_{i}\right) L_{d j}\left(x_{i}\right) \\
& t\left(x_{i}\right)=\frac{T_{B}\left(x_{i}\right)}{\sum_{j=1}^{k}\left(\lambda_{i}(j) \zeta_{j}^{\text {staff }}\right)} \cdot+\max \left(\lambda_{i}(j) \cdot\left(T_{R_{j}}\left(x_{i}\right)+T_{D_{j}}\left(x_{i}\right)\right)+T_{i j}\right)_{j=1,2, \ldots, k}, \\
& \lambda_{i}(j)= \begin{cases}0, & \text { repair team } j, \\
& \text { refuses the task } i, \\
& \text { repair team } j, \\
1, & \text { accepts the task } i,\end{cases} \\
& T_{R}=\left\{\begin{array}{l}
0, \quad \zeta^{\text {resource }} \text { astisfies the needs, } \\
\sum_{\Gamma} \frac{\omega^{T}}{60}, \zeta^{\text {resource }} \text { unastisfies the needs } \\
T_{D}=\sum_{\Gamma} \frac{\omega^{T}}{60},
\end{array}\right.
\end{aligned}
$$

where $T_{R}$ is the repair resource allocation time, $T_{D}$ is the drive time to the failure point, $T_{B}$ is the standard estimated working time of repair task, $T_{i j}$ is the total time spent by repair team $j$ before executing the task $i$, and $\lambda_{i}(j)$ is a repair decision variable which indicates whether the repair team $j$ executes the task $i$. The value of $\lambda_{i}(j)$ is obtained by the roulette selection method through evaluating the resource demand of repair task, execution capability, and resource allocation of the repair team, and its specific details are included in [19]. $\omega_{d j}$ is the weight coefficient of the load in class $d j ; L_{d j}\left(x_{i}\right)$ is the outage power of the load in class $d j$ that is restored after the faulty component $x_{i}$ is repaired. The 
system has $m$ failure components, $n$ operable switches, and $k$ repair teams in total. $\zeta_{j}^{\text {staff }}$ is the operation ability factor of the repair team $j$.

Solve the repair strategy optimization model with the discrete bacterial colony chemotaxis algorithm (DBCC) to obtain the sequence of repair tasks [20], where the control variable $x_{i}$ needs to be discretized:

$$
x_{i}=\frac{a_{i}}{\sum_{i=1}^{m+n} a_{i}},
$$

where $a_{i}$ is a random number between 0 and 1 and represents the fault point label of $x_{i}$. The processed $x_{i}$ is no longer the label of the faulty component but represents the position of the bacteria in the solution space. Rearrange $x_{i}$ from small to large, and then the serial number of $x_{i}$ in the new sequence represents the order in which the emergency repair is performed. According to the corresponding label $a_{i}$ of $x_{i}$, the fault point label corresponding to the emergency repair sequence can be known.

\subsubsection{Constraints}

(1) Power flow constraints:

$$
\begin{aligned}
\sum_{(i, j) \in \Omega_{D}} P_{i j, t}-\sum_{(j, i) \in \Omega_{D}} P_{j i, t} & =P_{i, t}^{G}-u_{i, t}^{L} x_{i, t} P_{i, t}, \quad \forall i \in \Omega_{L}, t \in \Omega_{T}, \\
\sum_{(i, j) \in \Omega_{D}} Q_{i j, t}-\sum_{(j, i) \in \Omega_{D}} Q_{j i, t} & =Q_{i, t}^{G}-u_{i, t}^{L} x_{i, t} Q_{i, t}, \quad \forall i \in \Omega_{L}, t \in \Omega_{T}, \\
x_{i, t} & \in\{0,1\}, \quad \forall i \in \Omega_{L}, t \in \Omega_{T}, \\
Q_{i, t} & =\tau_{i}(t) Q_{i}^{L B}, \quad \forall i \in \Omega_{L}, t \in \Omega_{T}, \\
0 & \leq P_{i, t}^{G} \leq P_{i}^{G, \max }, \quad \forall i \in \Omega_{L}, t \in \Omega_{T}, \\
0 & \leq Q_{i, t}^{G} \leq Q_{i}^{G, \max }, \quad \forall i \in \Omega_{L}, t \in \Omega_{T}, \\
\left|P_{i j}\right| & \leq P_{i j \max }, \quad \forall(i, j) \in \Omega_{D}, \\
\left|Q_{i j}\right| & \leq Q_{i j \max }, \forall(i, j) \in \Omega_{D},
\end{aligned}
$$

where $\Omega_{\mathrm{D}}$ is the set of branch and $P_{i j}$ and $Q_{i j}$ represent the active and reactive power flowing from the distribution network nodes $i$ to $j$, respectively. $Q_{i, t}$ is the reactive power of the distribution network node $i$ at the time $t . P_{i, t}^{G}$ and $Q_{i, t}^{G}$ represent the active and reactive power output of the generation at distribution network node $i$ when the time is $t$, respectively. $x_{i, t}$ is a $0-1$ variable which represents the power supply states of the distribution network node $i$ at the time $t\left(x_{i, t}=1\right.$, the node $i$ has power supply; $x_{i, t}=0$, the node $i$ is in power-off). $P_{i}^{G, \max }$ and $Q_{i}^{G}, \max$ represent the maximum active and reactive power output of the generation at distribution network node $i$, respectively. $P_{i j \max }$ and $Q_{i j \max }$ are the maximum capacity of active and reactive power flowing from the distribution network node $i$ to $j$.

(2) Voltage constraints: The voltage of each node cannot exceed the limit during the repair process and switching operation.

$$
U_{\min } \leq U \leq U_{\max }
$$

(3) Switching constraints: Taking the switch under operating as a virtual fault point, the repair time is the corresponding switching time, and the switching action is restricted by the principle of gird reconfiguration. The switch state remains unchanged between two repair tasks of the virtual fault point [20]. Besides, the switch will no longer change its state when all loads have been restored to power.

\section{Resilience Evaluation}

4.1. Indices. Different from reliability, resilience has its newly developing and emerging concept in the area of power systems; it refers to the ability to absorb and rapidly recover from an extraordinary disturbance event [33]. Taking the load performance to represent system performance, Figure 2 shows a conceptual resilience trapezoid, which clearly demonstrates the stages of a power system associated to an external disturbance [34].

In Figure 2, $F_{0}$ is the original state of the system; the disturbance hits the system at $t_{0}$, which results in the system performance deteriorating; $t_{1}$ is the maximum loss moment of the system and $F_{1}$ is the corresponding performance level of the system. Following the disturbance, the system will reside in the postdisturbance self-recovery state $\left(t_{1}-t_{2}\right)$, where grid reconstruction measures would be taken to restore the power supply in the nonfaulty areas; $F_{2}$ is the system performance level after reconstruction. Then the grid repair workers apply proper and effective repair measures to make the system fully recover from the disturbance $\left(t_{2}-t_{3}\right)$ and the system performance level will eventually return to $F_{3}$ (the postdisturbance system performance level can be different from the original state). 


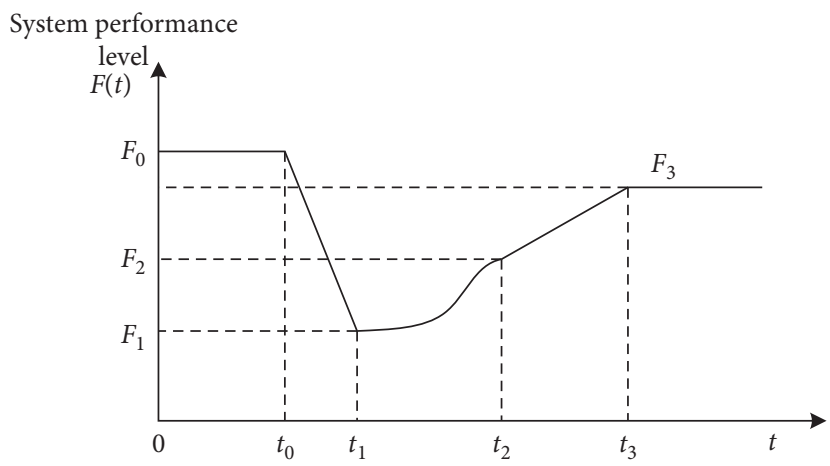

Figure 2: The power system resilience trapezoid model.

Based on the trapezoid model, several different indicators have been put forward to quantitatively analyze the system resilience. These resilience metrics can be divided into two categories in terms of their composition: single indices and composite indices. The single index, such as the integral of the system performance function from the beginning of the disturbance $\left(t_{0}\right)$ to the complete restoration of the system $\left(t_{3}\right)$ or the proportion of system function maintaining the normal state during extreme disasters, is an assessment for the overall process $[3,35]$. While the composite index is used to propose several evaluation indices for each stage or capability of resilience and then integrate them through a weighted combination, such as the combination of robustness, rapidity, and performance loss, evaluating resilience from different perspectives [36]. In this paper, considering the impact on system resilience of the complicated coupling relationship of distribution-traffic-human network is mainly reflected in the recovery time, the distribution network resilience $R$ is defined as the time it takes for the system to recover to $95 \%$ of its original state after being hit by the extreme weather event, and the $95 \%$ of the original state is named the recovery state. To further evaluate the performance of system resilience during the repair process after disasters, the following resilience indexes of the repair process of the distribution network are proposed, which are for a certain disaster scene.

(1) Adaptive rate R1: The probability that the system performance meets the minimum performance requirements and the recovery time does not exceed the longest recovery time limit. The minimum system performance value is $50 \%$ of the total system power and the longest recovery time is determined according to the social importance of the load.

$$
R_{1}=P(\min (F(t)>\underline{f}, t<\bar{t})) .
$$

(2) Repair rate R2: The recovery speed at which the system can restore to a stable state after the disaster.

$$
R_{2}=\frac{F_{3}-F_{1}}{t_{3}-t_{1}}
$$

(3) Economic loss rate R3: The ability of the system to decrease economic loss after the disaster.

$$
\begin{aligned}
& R_{3}=\frac{M_{\Delta}}{M_{0}} \\
& M=\sum_{i=1}^{m+n} e_{x_{i}, k}^{\text {loss }}\left(t\left(x_{i}\right)\right) L\left(x_{i}\right), \quad k=1,2,3,4,5,
\end{aligned}
$$

where $e_{x_{i}, k}^{\text {loss }}(t)$ is the economic loss caused by the fault point $x_{i}$ of load type $k$ with the outage time $t$ and $M_{\Delta}$ and $M_{0}$ both represent the economic loss value caused by continuous outages of various loads in the whole repair process; the former adopts the optimization repair strategy in calculation and the latter adopts the same repair sequence but only one repair team to execute all repair tasks, whose repair time for each fault task is the standard repair work time. The load type is divided into 5 categories: industry, business, medicine and health, government, and public utilities. The economic loss function of each type of load is shown in Figure 3 [37].

4.2. Resilience Enhancement Procedure. The resilience enhancement procedure of the complex distribution network under a hurricane disaster coupling with human resources and traffic network is shown in Figure 4.

\section{Results and Discussion}

In this section, we perform numerical experiments on a IEEE33 bus distribution system. The test system is fitted into an area that covers the range of latitude $\left(28.725^{\circ} \mathrm{N}-29.125^{\circ} \mathrm{N}\right)$ and longitude $\left(95.2^{\circ} \mathrm{W}-95.5^{\circ} \mathrm{W}\right)$, for demonstration purposes only. There are 37 lines, 5 contract switches, and 33 nodes in the primary network, as shown in Figure 5. The system voltage level is at $12.66 \mathrm{kV}$ and the total load is $3775 \mathrm{~kW}+\mathrm{j} 2300 \mathrm{kvar}$ [38].

According to the different social importance of each load, the loads are divided into 3 levels and given corresponding weight coefficients and repair time limits to assess the outage loss and the adaptive rate, as shown in Table 1. The load types of each node are shown in Table 2 for evaluating the economic loss. The human resources of emergency repair include repair teams and repair resource reserve, which impact the distribution network resilience. To 


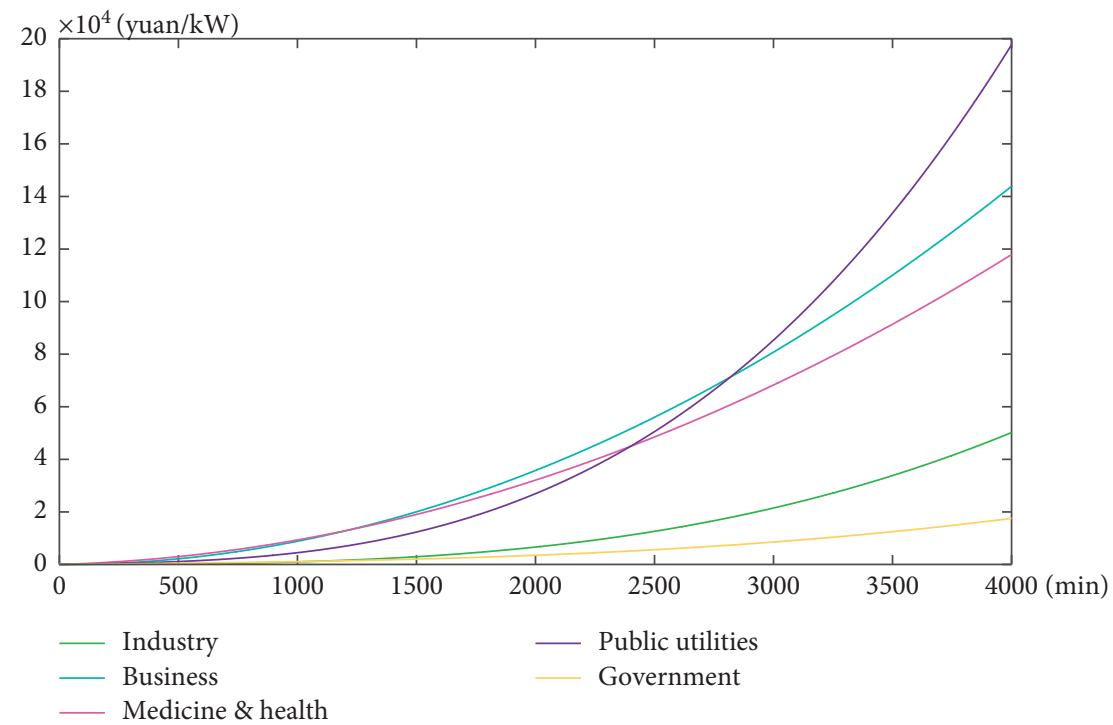

Figure 3: The economic loss function of each load type.

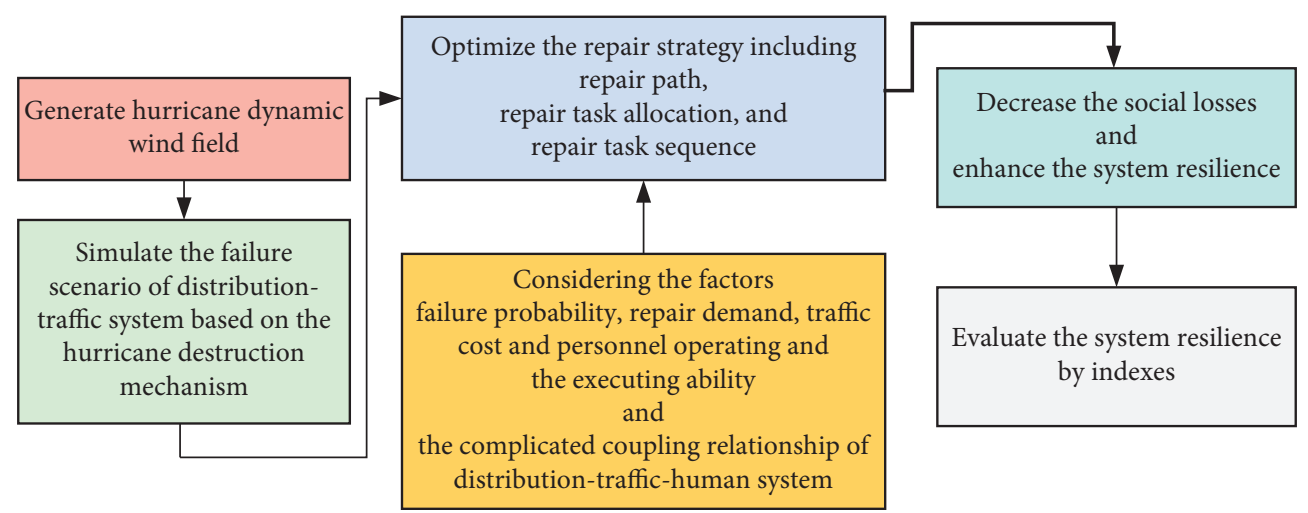

Figure 4: The process of enhancing the complex distribution network resilience coupling with human resources and traffic network under disasters.

consider these effects, it is assumed that the repair teams are stationed at nodes 1,7 , and 12, and the repair resource reserves are placed at nodes 10 and 11 , both in the traffic network. The structure of the complicated distributiontraffic-human coupling system is shown in Figure 6.

5.1. Simulation. For illustration, it is assumed that the hurricane lands at the location with latitude $28.9^{\circ} \mathrm{N}$ and longitude $95.2^{\circ} \mathrm{W}$. The hurricane is assumed to be moving with a translational speed of $12.5 \mathrm{mph}$ in the direction of $150^{\circ}$. The test system will be affected by the hurricane for 5 hours. Figure 7 shows the surface wind speed variations of the test system as the hurricane travels along its track with the sample interval of 30 minutes. It is clear to see that the wind speed at most nodes reaches its maximum at the first sample point and then declines over time. Figure 8 shows the specific wind speed of the test system when $T=30 \mathrm{~min}$.

Since the damaged state of the complicated distributiontraffic network is related to the suffered wind speed, use the maximum wind speed of each node suffered during the hurricane to calculate the component failure probability, by (1) to (5). The results are shown in Figure 9. Sample the failure events based on failure probability, and the failure scenario of distribution network is obtained as shown in Figure 10. The failed lines are L3-4, L4-5, L10-11, L11-12, and L12-13; the failed switches are installed at L10-11, L1112, L12-13, and L20-21; the transformer at node 12 is completely damaged and needs to be replaced; the line L2021 is broken but still repairable; and the nonfault nodes N69, N14-17, and N25-32 are affected to outage. Table 3 shows the parameters of each failure event, including the repair resource requirement, the standard repair time, and the load level [39].

5.2. Analysis of the Repair Strategy. There are 3 repair teams in this case and the repair capabilities of each team are shown in Table 4.

The repair capability parameters indicate that none of the single repair teams could meet the resource requirement of failure node N12; and the failed lines L10-11 and L20-21 


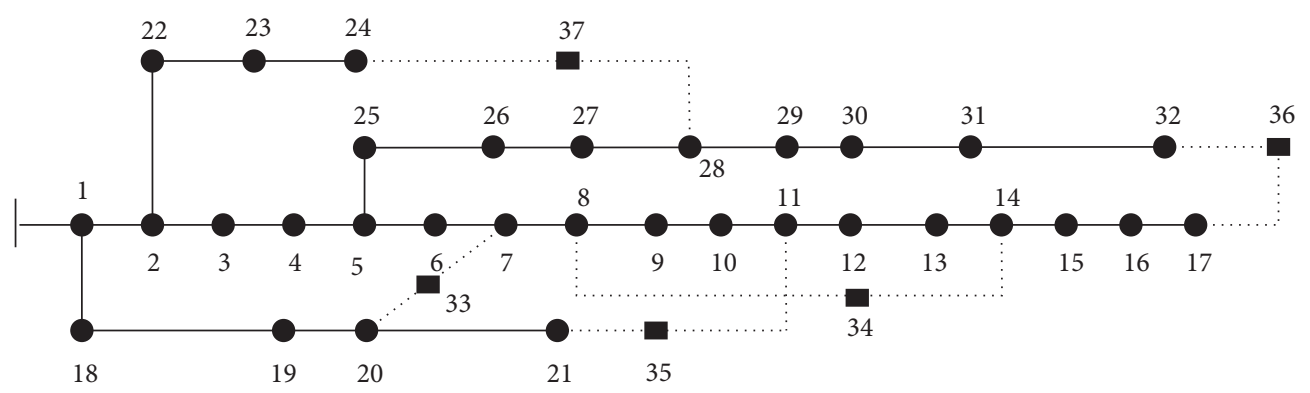

Figure 5: The 33-bus distribution network.

TABle 1: The load level parameters.

\begin{tabular}{lccc}
\hline Level & Node number & The weight coeffcient & $\bar{t} / \mathrm{h}$ \\
\hline 1 & $2,3,4,7,8,9,15,16,17,28,29$ & 900 & 24 \\
2 & $1,6,10,11,12,21,24,26,27$ & 30 & 40 \\
3 & $5,13,14,18,20,22,23,25,30,31,32,33$ & 1 & 60 \\
\hline
\end{tabular}

TABLE 2: The load type parameters.

\begin{tabular}{lc}
\hline Type & Node number \\
\hline Industry & $1,6,24,26$ \\
Business & $10,11,12,21,27$ \\
Medicine \& health & $2,3,4,16,17$ \\
Government & $7,8,9,15,28,29$ \\
Public utilities & $5,13,14,18,20,22,23,25,30,31,32,33$ \\
\hline
\end{tabular}

require a large number of repair resources of which only repair team 1 meets the needs alone. As to repair teams 2 and 3 , they need to cooperate or allocate more resources from the reserve point to complete the repair tasks. Considering the repair capability of repair teams and the characteristics of failure events, the repair task assignment scheme is obtained based on the utility value (see Table 5), as shown in Table 6 [19].

It can be noted that, due to the requirements of the repair capability and repair resources, the repair task of node N12 prefers to be assigned to repair teams 1 and 2, followed by repair teams 1 and 3 . And to make the high-level load online L3-4 restore as soon as possible, the repair task of line L3-4 prefers to be assigned to repair team 1 alone. The repair task of line L10-11 has never been assigned to repair team 3 due to the resource requirements and geographic distance. While the repair task of line L20-21 prefers to be completed by repair team 3 in cooperation with others for the same reason.

Optimizing the operation sequence of repair plans to minimize the social losses caused during the entire emergency repair process, 3 better repair plans are obtained for reference of the grid workers. Table 7 shows the optimized emergency repair plan, including the repair sequence, the social loss value, and the repair time. Figure 11 particularly demonstrates the social loss variations of each repair plan during the entire emergency repair stage. In order to further illustrate the emergency repair process, take plan 2 as an example to demonstrate the repair sequence, repair team, and the social loss of each repair task, as shown in Figure 12.
As a supplement, Figure 13 shows the optimized repair paths of repair team 1 in plan 2. The contribution of the repair teams in each repair task of plan 2 is shown in Figure 14, quantified by the social loss.

It is observed that the switch operation is always executed first in every scheme. This is not only because nodes 36 and 37 are virtual fault points whose repair time is just the response time of switch action, but also the new grid formed by the change of switch state can restore the maximum power supply of the nonfault nodes. The optimized scheme conforms to the rule that the grid reconstruction measures are prioritized during the emergency repair process of distribution network.

Figure 15 shows the resilience and load variations of the test system adopted the repair plans. Evaluate the system resilience under the 3 repair plans by the proposed indexes, and the results are shown in Table 8 . It can be noticed that the performances of adaptive rate $R_{1}$ and repair rate $R_{2}$ are both related to the repair time. The shorter the repair time, the better the index performance, which means indexes $R_{1}$ and $R_{2}$ are both negatively correlated with the resilience value $R$. While the economic loss rate $R_{3}$ is relatively independent of the resilience $R$ because the different load types have a significant difference in economic loss.

Choose repair plan 3 as an example to demonstrate the relationship between system resilience $R$ and repair rate $R_{2}$, which has the best resilience performance. Other parameters remain unchanged, only the operation ability of the repair personnel is gradually increased during the simulation process (a total increase of $30 \%$, with an increase of $5 \%$ each time), and the curve of the resilience $R$ and repair rate $R_{2}$ is obtained as shown in Figure 16. Besides, the resilience improvement and shortening of repair time brought about by the increase in repair rate $R_{2}$ will also increase the adaptive rate $R_{1}$ and reduce the economic loss rate $R_{3}$ of the test system, as shown in Figure 17.

It is observed that as the repair rate $R_{2}$ increases and accumulates to a certain extent, the adaptive rate $R_{1}$ of the 


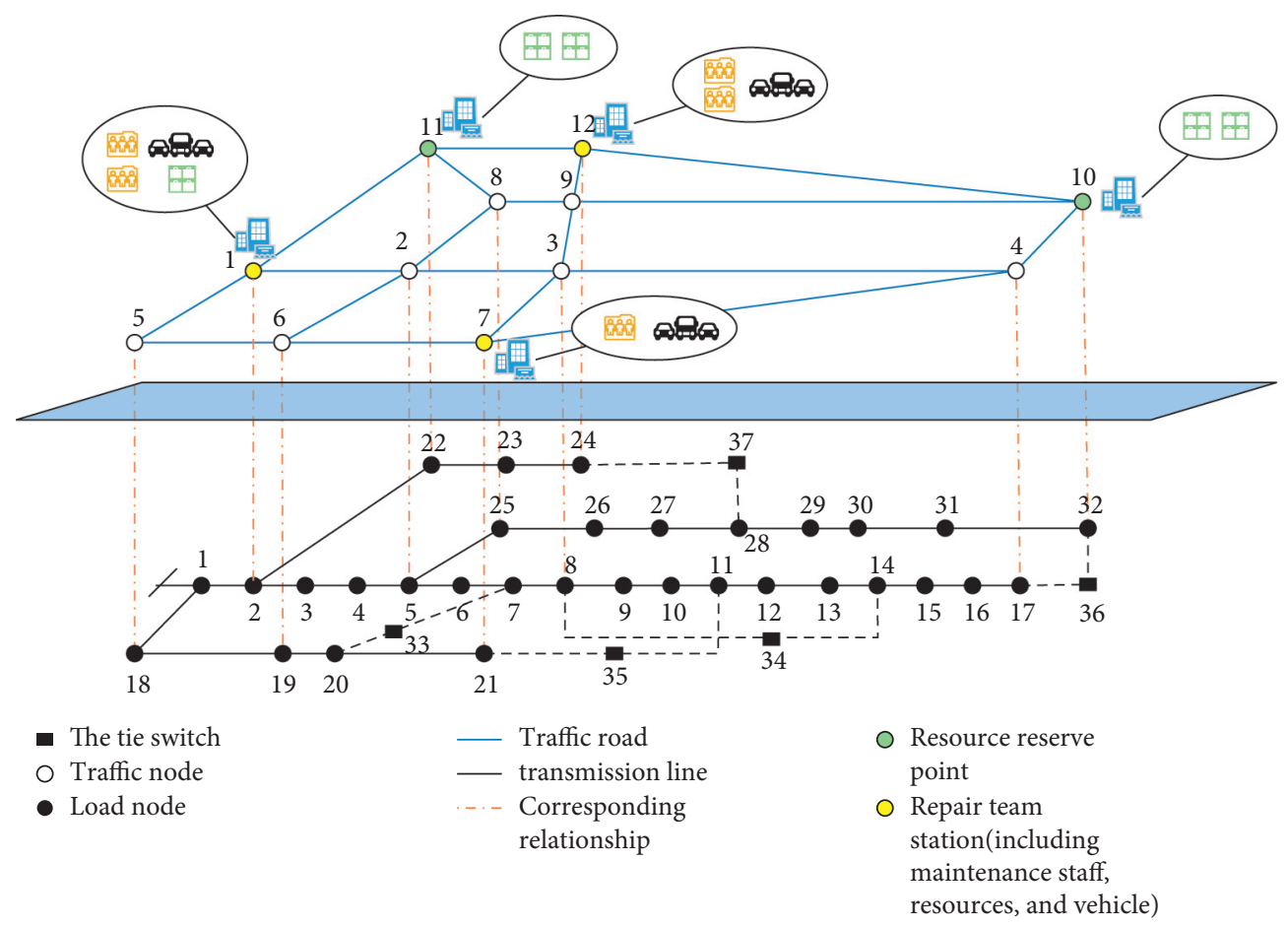

FIGURE 6: Model of the complicated distribution-traffic-human coupling system.

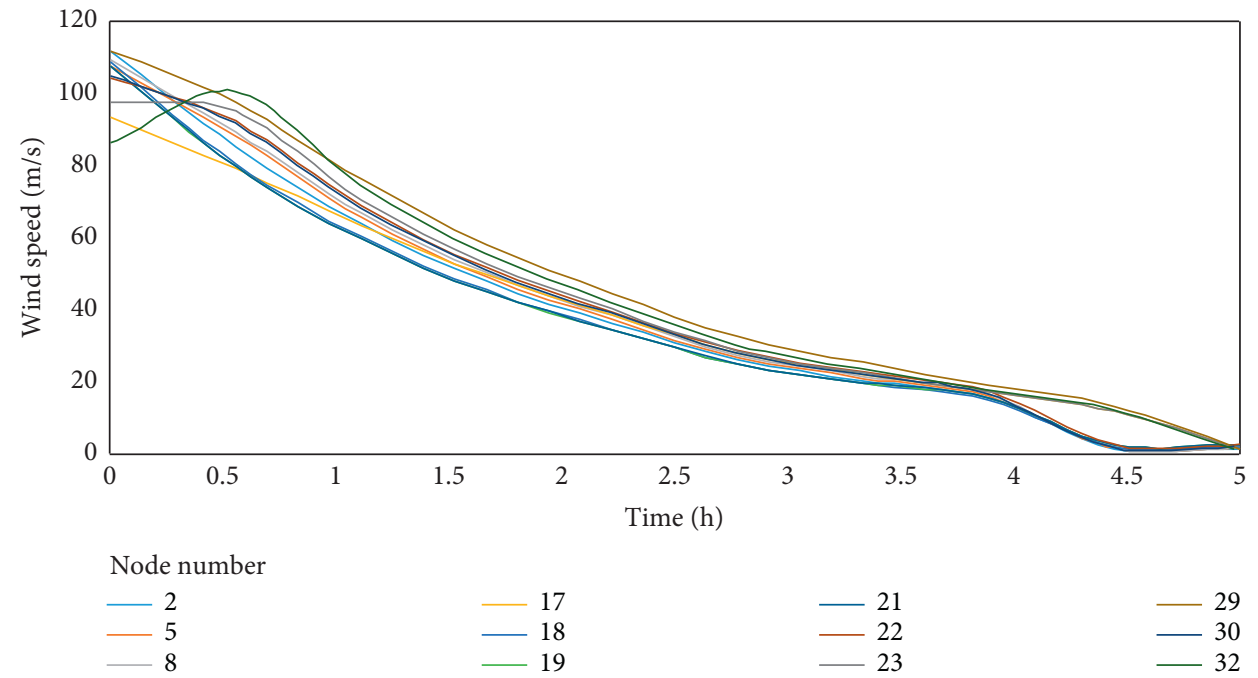

Figure 7: The wind speed attenuation curve. Choose the nodes which are in both the traffic network and the distribution network as a representative, and the node number is corresponded to the distribution network.

system rises stepwise due to the characteristic of the probability. And curves of the $R-R_{2}$ and $R_{3}-R_{2}$ have a similar trend which shows that the higher the quality of the human resources involved in the emergency repair process, the higher the corresponding system repair rate $R_{2}$, which improves the system resilience and decreases the economic loss. However, the continuous decrease of the slope of the curves indicates that the improved efficiency will gradually level off as the repair rate continues to increase. This is because resilience is related to the time-consuming repair process consisting of the repair task execution time and driving time. Improving the operation ability of the repair 


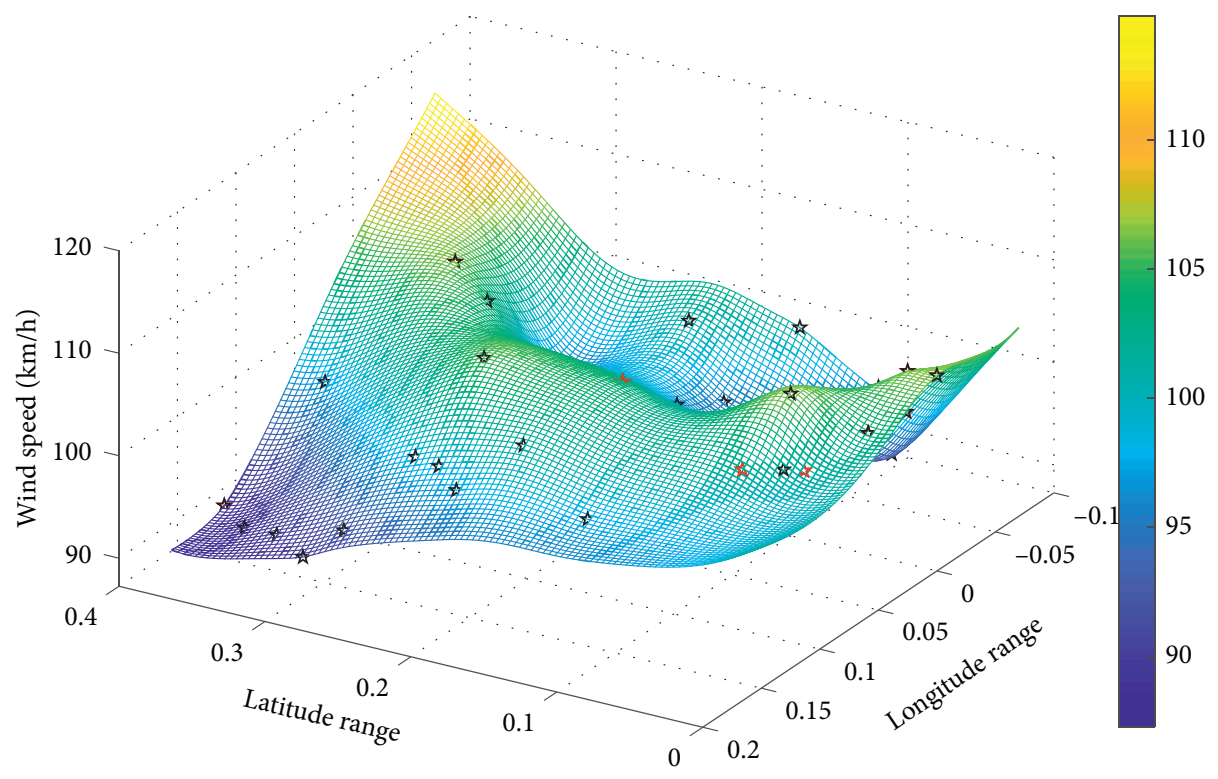

th Traffic node

it Distribution node

Figure 8: The wind speed at each node of system $(T=30 \mathrm{~min})$.

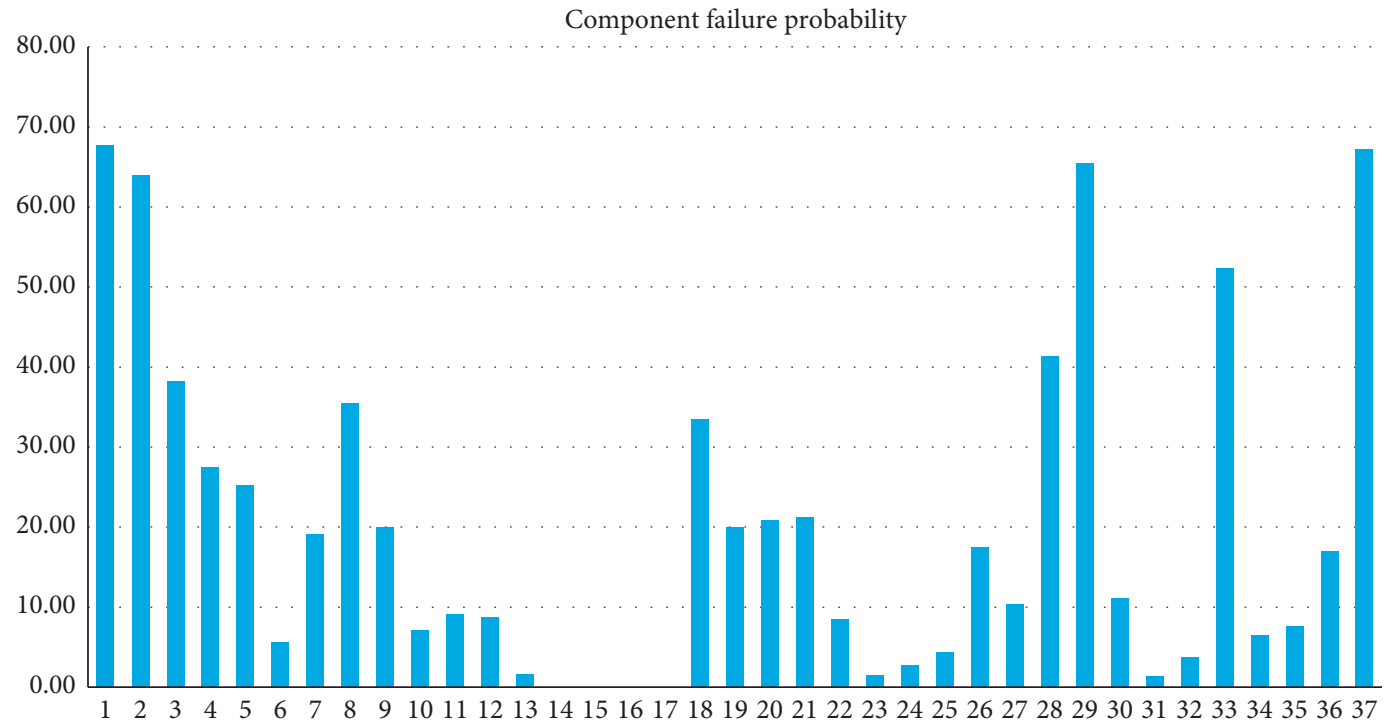

Figure 9: Component failure probability of the distribution network. The $Y$-axis is the failure probability, and the $X$-axis is the node label of IEEE33 bus distribution system.

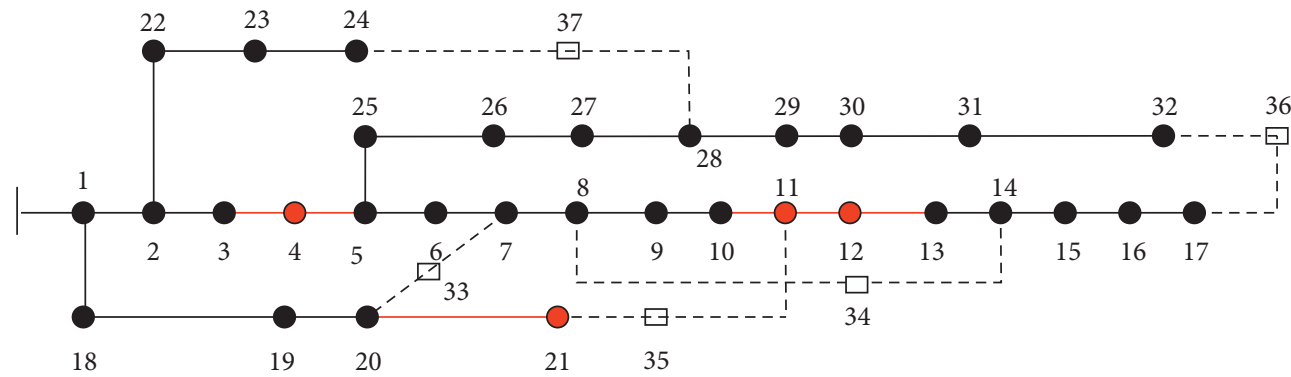

FIgURE 10: The distribution network failure scenario. 
TABLE 3: Fault event set.

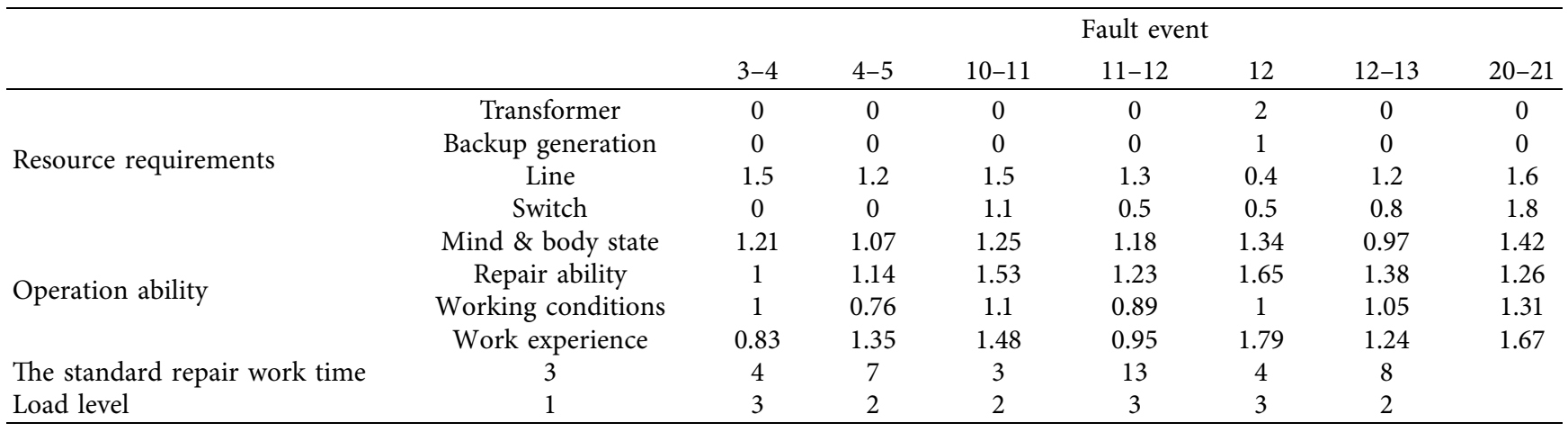

TABLE 4: Repair team parameters.

\begin{tabular}{|c|c|c|c|c|}
\hline \multicolumn{2}{|c|}{ Repair team } & 1 & 2 & 3 \\
\hline \multirow{4}{*}{ Resource requirements } & Transformer & 1.5 & 0.5 & 1 \\
\hline & Backup generation & 1 & 0.4 & 0.8 \\
\hline & Line & 1.8 & 1.5 & 1.3 \\
\hline & Switch & 2 & 0.8 & 1.4 \\
\hline \multirow{4}{*}{ Operation ability } & Mind \& body state & 1.25 & 1.32 & 1.65 \\
\hline & Repair ability & 1.57 & 1.46 & 1.87 \\
\hline & Working conditions & 0.91 & 1 & 1.07 \\
\hline & Work experience & 1.68 & 0.89 & 1.93 \\
\hline Station node & 1 & 12 & 7 & \\
\hline
\end{tabular}

TABle 5: The $u_{i j}$ value of repair teams to each fault event.

\begin{tabular}{|c|c|c|c|c|c|c|}
\hline \multirow{2}{*}{ Fault event } & \multicolumn{6}{|c|}{ Repair team } \\
\hline & 1 & 2 & 3 & $1+2$ & $2+3$ & $1+3$ \\
\hline $3-4$ & 0.95 & 0.65 & 0 & 0.40 & 0.38 & 0.37 \\
\hline $4-5$ & 0.85 & 0.63 & 0.53 & 0.42 & 0.40 & 0.36 \\
\hline $10-11$ & 0.74 & 0 & 0 & 0.49 & 0.54 & 0.46 \\
\hline $11-12$ & 0.64 & 0.65 & 0.52 & 0.33 & 0.36 & 0.31 \\
\hline 12 & 0 & 0 & 0 & 0.89 & 0.65 & 0.71 \\
\hline $12-13$ & 0.60 & 0.65 & 0.65 & 0.42 & 0.45 & 0.37 \\
\hline $20-21$ & 0.62 & 0 & 0 & 0.67 & 0.75 & 0.86 \\
\hline
\end{tabular}

TABLE 6: Task assignment scheme set.

\begin{tabular}{lccc}
\hline Plan & \multicolumn{2}{c}{ Repair team } & 3 \\
\hline 1 & $3-4,4-5,10-11,12,20-21$ & $10-11,12,12-13,36$ & $11-12,20-21,37$ \\
2 & $3-4,4-5,10-11,12,37$ & $10-11,11-12,12,20-21$ & $12-13,20-21,36$ \\
3 & $3-4,10-11,12,20-21,36$ & $4-5,11-12,12,37$ & $12-13,20-21$ \\
4 & $3-4,4-5,10-11,12,36$ & $11-12,12-13,20-21$ & $12,20-21,37$ \\
5 & $10-11,12,20-21,37$ & $3-4,11-12,36$ & $4-5,12,12-13$ \\
6 & $10-11,12,20-21$ & $3-4,12,36,37$ & $4-5,11-12,12-13$ \\
\hline
\end{tabular}

TABLE 7: Emergency repair sequence and social loss.

\begin{tabular}{|c|c|c|c|c|c|}
\hline \multirow{2}{*}{ Plan } & \multicolumn{3}{|c|}{ Repair team } & \multirow{2}{*}{ Social loss $(\mathrm{kWh})$} & \multirow{2}{*}{ Repair time (h) } \\
\hline & 1 & 2 & 3 & & \\
\hline 2 & $37,3-4,10-11,12,4-5$ & $10-11,12,11-12,20-21$ & $36,12-13,20-21$ & 9631761 & 63.26 \\
\hline 3 & $36,3-4,12,20-21,10-11$ & $37,4-5,12,11-12$ & $12-13,20-21$ & 9198327 & 47.83 \\
\hline 5 & $37,10-11,12,20-21$ & $36,3-4,11-12$ & $4-5,12,12-13$ & 8966593 & 53.15 \\
\hline
\end{tabular}




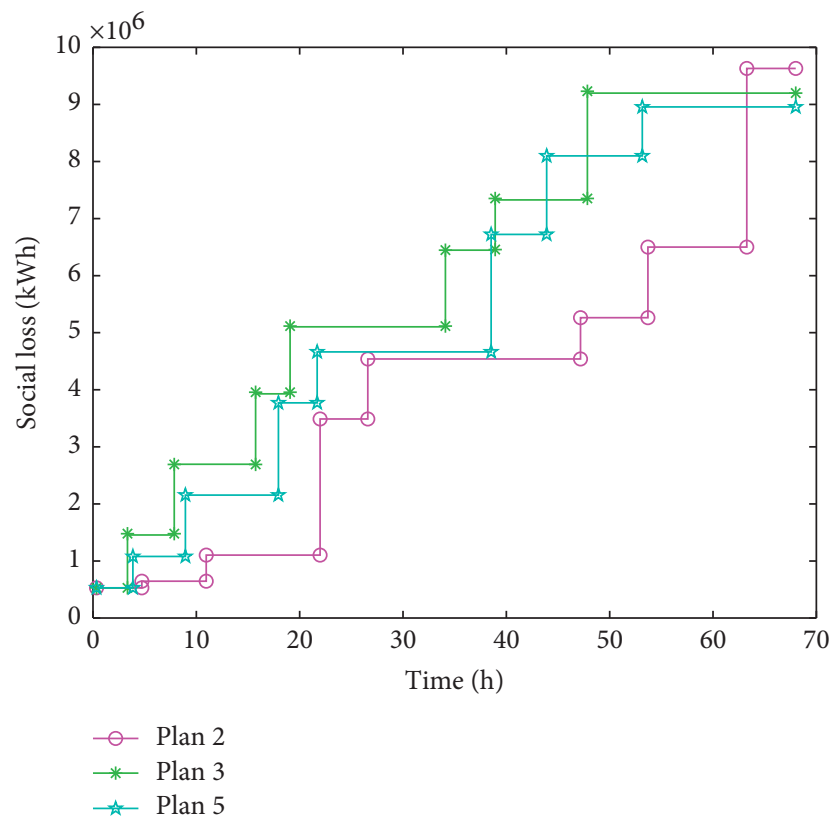

Figure 11: The social loss variations of the test system.

Plan 2

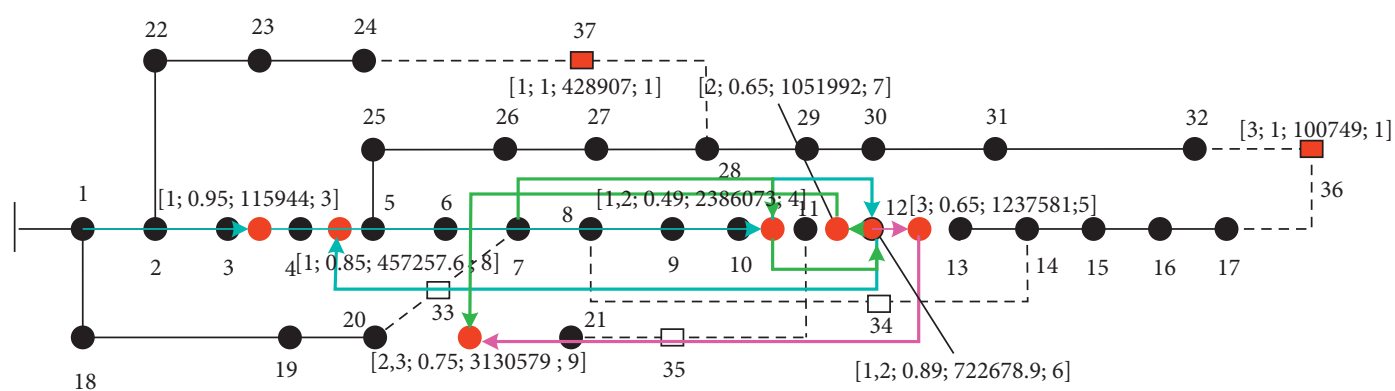

$[a, \zeta, b, c]$

$a:$ repair team number

$b$ : social loss value

$c$ : repair sequence

$\zeta$ : repair capability rate

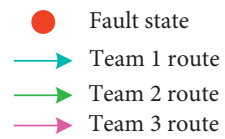

FIgURE 12: The emergency repair process of plan 2.

Plan 2

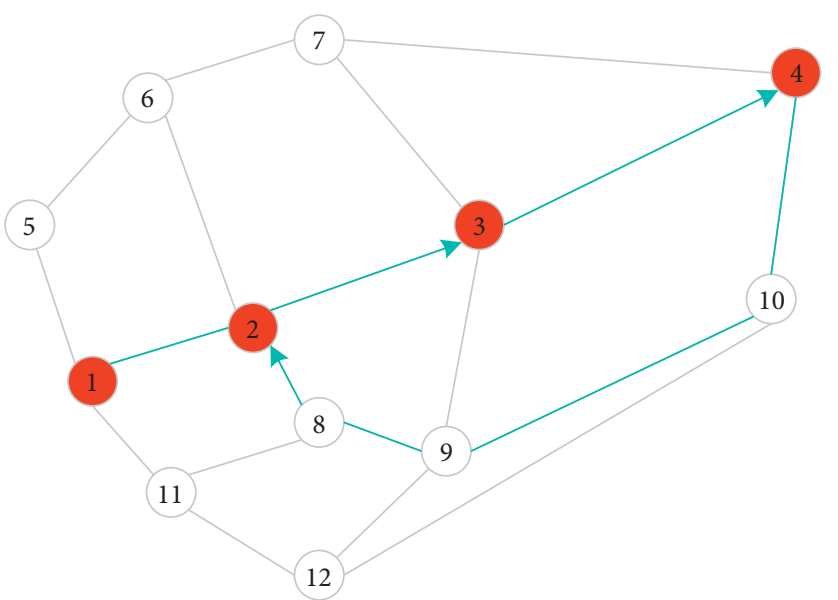

Available path

(i) Traffic node

$\rightarrow$ Team 1 route

Fault node

FIGURE 13: The results of repair path planning. 


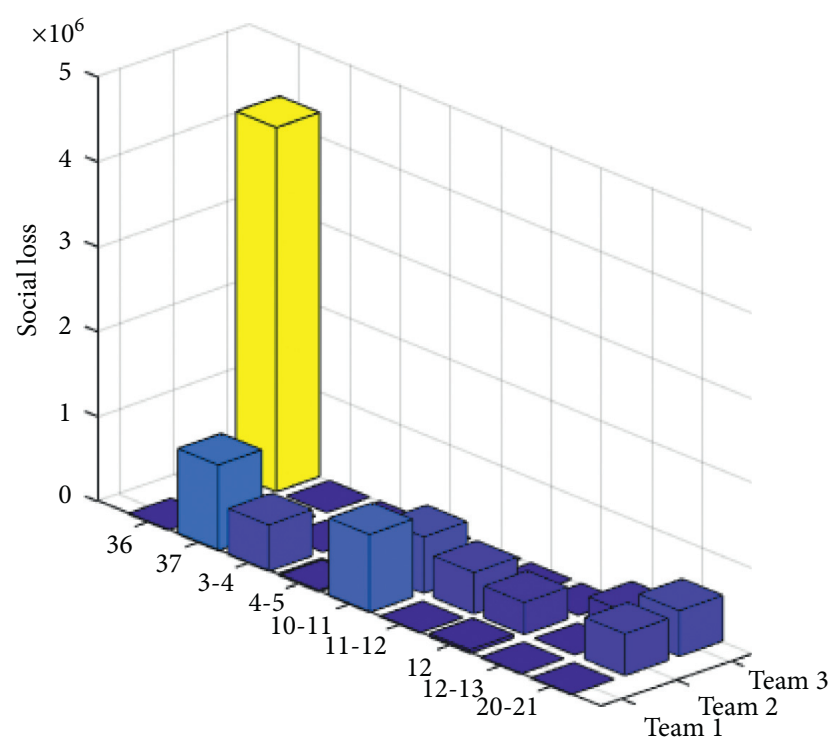

Plan 2

Figure 14: The contribution of repair teams in each repair task.

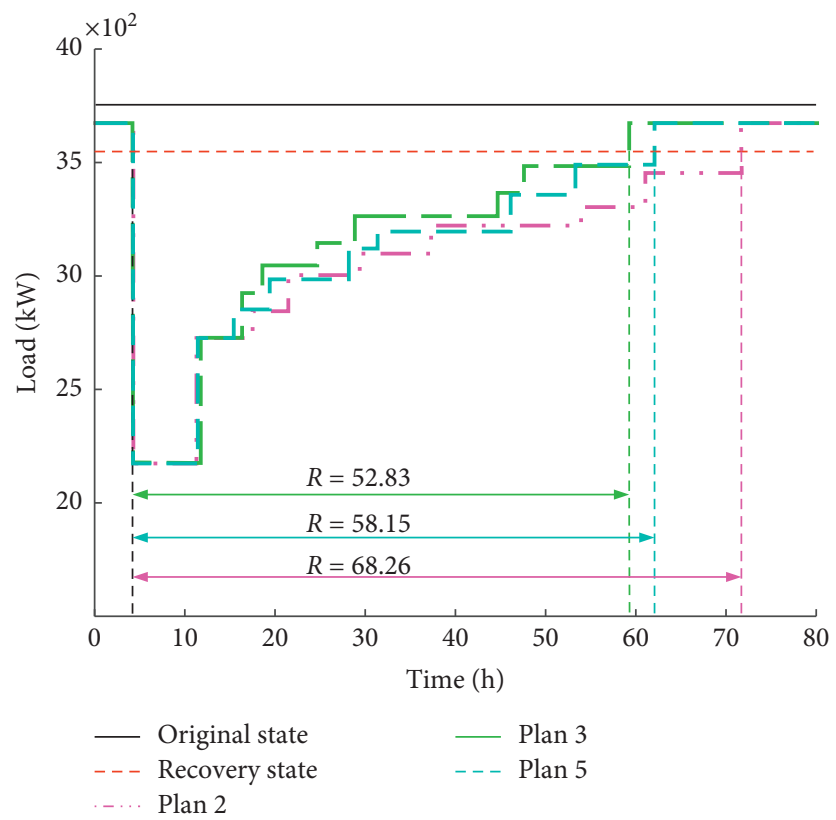

Figure 15: The resilience and load variations of the test system.

TABLE 8: Distribution network resilience index of different repair plans.

\begin{tabular}{lcccc}
\hline Plan & $R$ & $R_{1}$ & $R_{2}$ & $R_{3}$ \\
\hline 2 & 68.26 & 97.11 & 179.32 & 80.15 \\
3 & 52.83 & 99.01 & 237.15 & 85.09 \\
5 & 58.15 & 98.12 & 213.43 & 81.52 \\
\hline
\end{tabular}




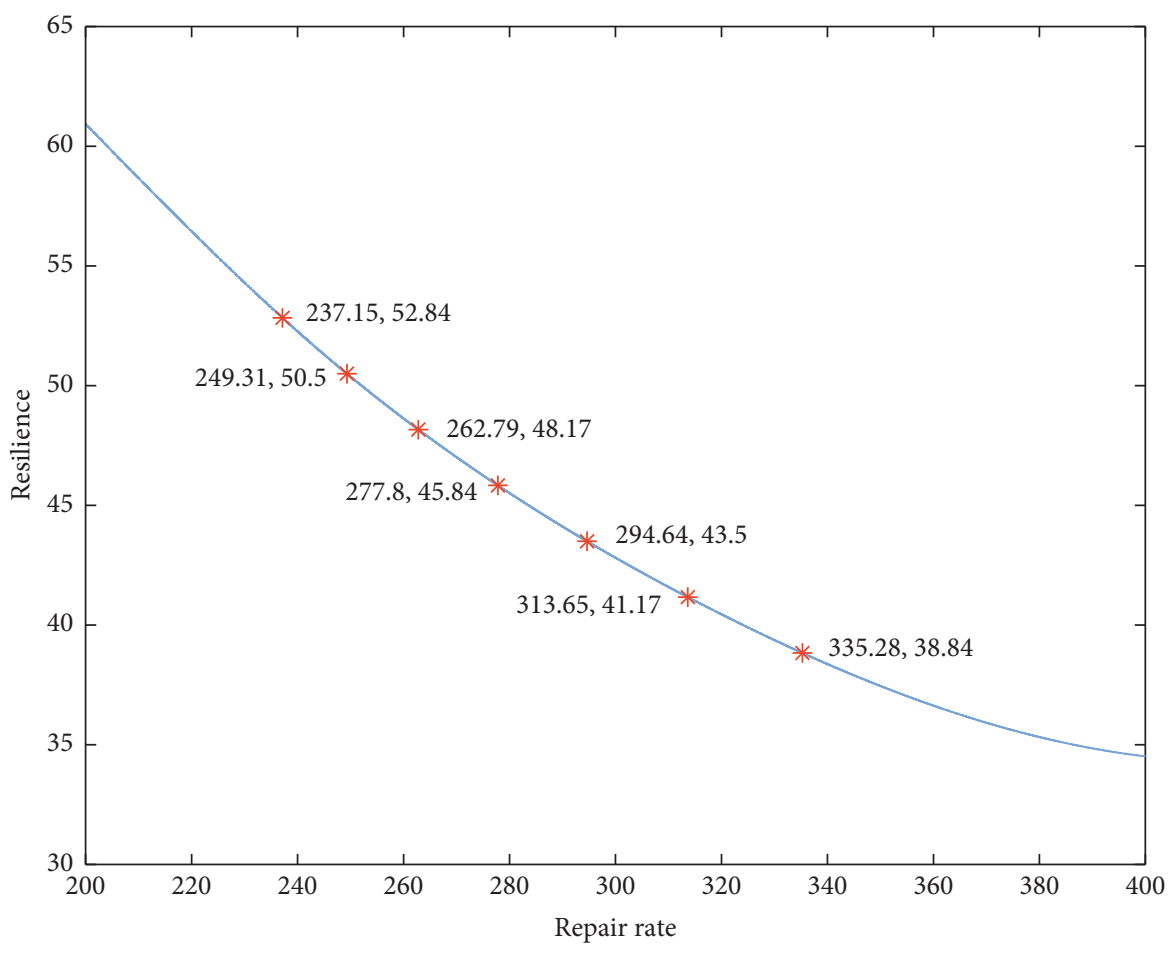

* $\left(R_{2}, R\right)$

FIgURE 16: The curve of resilience and repair rate.

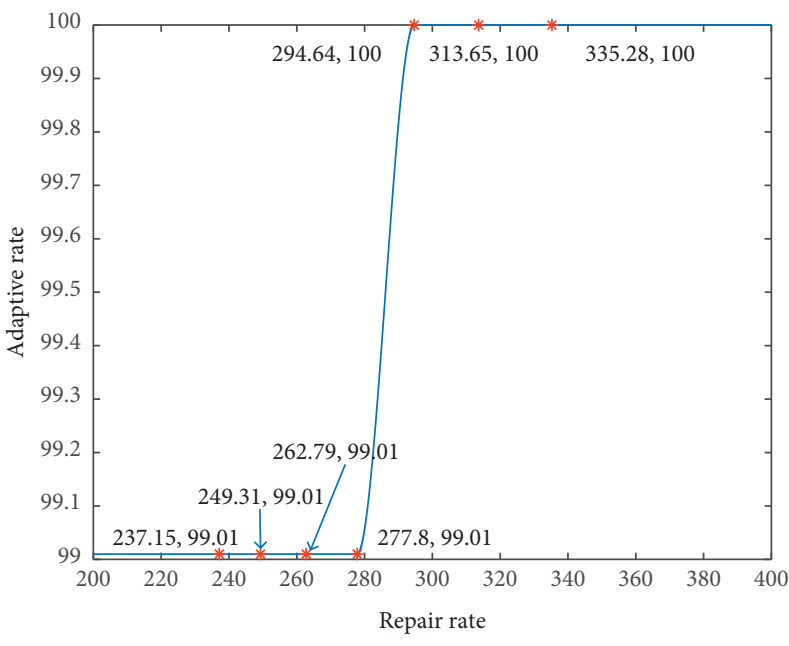

* $\left(R_{2}, R_{1}\right)$

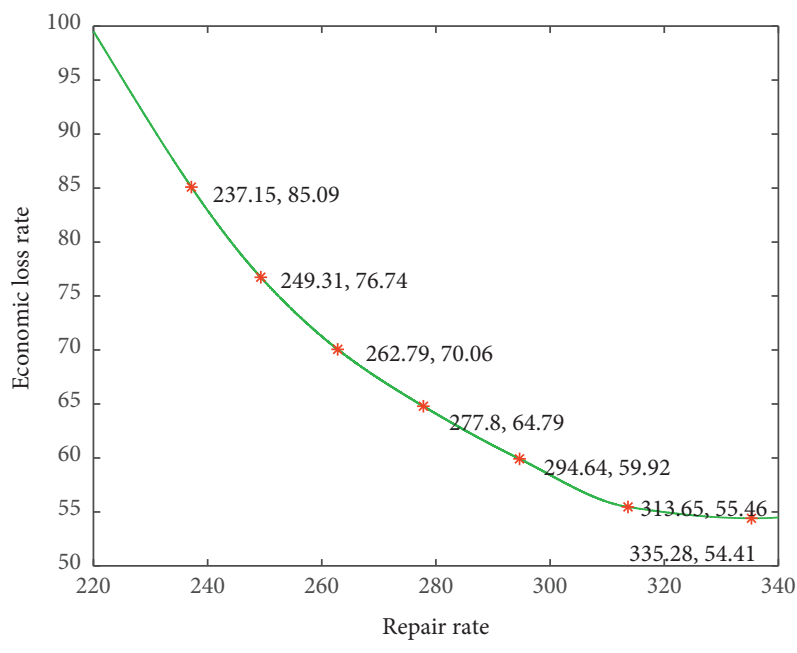

* $\left(R_{2}, R_{3}\right)$

(a)

(b)

Figure 17: The curve of three resilience evaluation indices.

personnel can quickly shorten the repair task execution time in the early stage, while it cannot reduce the repair time without limitation.

\section{Conclusions}

Considering the complex coupling relationship between distribution network, traffic network, and human resources in the emergency repair stage, this paper presents an optimization method to improve the resilience of the complicated distribution-traffic-human coupling system against extreme weather events. Tested on the IEEE33 bus system, the numerical results show that the repair plans, obtained by the proposed model, shorten the execution time of the emergency repair plans and decrease the social and economic losses caused by outages, thereby enhancing the 
complex distribution network resilience. The results of evaluation indexes show that the resilience performance is positively correlated with the system adapt and repair abilities. Moreover, improve the quality of human resources by enhancing the operation ability of repair personnel can speed up the system repair rate and increase the system resilience effectively.

\section{Data Availability}

The data used to support the findings of this study are available within the article.

\section{Conflicts of Interest}

The authors declare that there are no conflicts of interest regarding the publication of this paper.

\section{Acknowledgments}

This work was supported in part by the National Natural Science Foundation of China under Grant no. 51767002.

\section{References}

[1] A. Arab, A. Khodaei, S. K. Khator, K. Ding, V. A. Emesih, and Z. Han, "Stochastic pre-hurricane restoration planning for electric power systems infrastructure," IEEE Transactions on Smart Grid, vol. 6, no. 2, pp. 1046-1054, 2015.

[2] H. Farzin, M. Fotuhi-Firuzabad, and M. Moeini-Aghtaie, "Enhancing power system resilience through hierarchical outage management in multi-microgrids," IEEE Transactions on Smart Grid, vol. 7, no. 6, pp. 2869-2879, 2016.

[3] M. Bruneau, S. E. Chang, R. T. Eguchi et al., "A framework to quantitatively assess and enhance the seismic resilience of communities," Earthquake Spectra, vol. 19, no. 4, pp. 733-752, 2003.

[4] S. Ma, L. Su, Z. Wang, F. Qiu, and G. Guo, "Resilience enhancement of distribution grids against extreme weather events," IEEE Transactions on Power Systems, vol. 33, no. 5, pp. 4842-4853, 2018.

[5] L. B. Chen and H. Chen, "Optimal planning of automatic switches in resilient distribution network against extreme disasters," Electric Power Automation Equipment, vol. 11, 2019.

[6] C. Wei, Z. Shen, D. Xiao, L. Wang, X. Bai, and H. Chen, “An optimal scheduling strategy for peer-to-peer trading in interconnected microgrids based on RO and Nash bargaining," Applied Energy, vol. 275, 2021.

[7] A. Gholami, T. Shekari, F. Aminifar et al., "Microgrid scheduling with uncertainty: the quest for resilience," IEEE Transactions on Smart Grid, vol. 7, p. 1, 2016.

[8] Q. A. Bie Zhaohong and Y. Lin, "Concept and research prospects of power system resilience," Automation of Electric Power Systems, vol. 39, no. 22, pp. 1-9, 2015.

[9] C. Y. GAO Haixiang, "Distribution systems resilience: an overview of research progress," Automation of Electric Power Systems, vol. 39, no. 23, pp. 1-8, 2015.

[10] X. Wang, M. Shahidehpour, C. Jiang, and Z. Li, "Resilience enhancement strategies for power distribution network coupled with urban transportation system," IEEE Transactions on Smart Grid, vol. 10, no. 4, pp. 4068-4079, 2018.
[11] C. Wei, M. Benosman, and T. Kim, "Online parameter identification for state of power prediction of lithium-ion batteries in electric vehicles using extremum seeking," International Journal of Control, Automation and Systems, vol. 17, no. 11, pp. 2906-2916, 2019.

[12] W. Wei, S. Mei, L. Wu et al., "Robust operation of distribution networks coupled with urban transportation infrastructures," IEEE Transactions on Power Systems, vol. 32, no. 3, pp. 2118-2130, 2017.

[13] S. Xie, Z. Hu, and J. Wang, "Two-stage robust optimization for expansion planning of active distribution systems coupled with urban transportation networks," Applied Energy, vol. 261, Article ID 114412, 2020.

[14] Y. Yang, W. Tang, Y. Liu, Y. Xin, and Q. Wu, "Quantitative resilience assessment for power transmission systems under typhoon weather," IEEE Access, vol. 6, no. 40, pp. 747-756, 2018.

[15] H. Gao, Y. Chen, S. Mei, S. Huang, and Y. Xu, "Resilienceoriented pre-hurricane resource allocation in distribution systems considering electric buses," Proceedings of the IEEE, vol. 105, no. 7, pp. 1214-1233, 2017.

[16] Y. Zhou, "Security analysis and evaluation method of distribution network cyber physical system (CPS) for network attack," Master's Thesis, Nanjing University Of Posts And Telecommunications, Nanjing, China, 2020.

[17] X. L. Liu and C. Yu, "Study on social factors in natural hazard risk management," Journal of Catastrophology, vol. 25, no. 4, pp. 120-126, 2010.

[18] M. Tavakoli and M. Nafar, "Human reliability analysis in maintenance team of power transmission system protection," Protection and Control of Modern Power Systems, vol. 5, no. 1, pp. 1-13, 2020.

[19] Z. J. Yang and J. Li, "A task allocation strategy of multifault rush repair for distribution network based on optimum utility," Transactions of China Electrotechnical Society, vol. 29, no. 6, pp. 263-270, 2014.

[20] Z. Liu and L. Yang, A rush repair strategy for distribution networks based on improved discrete multi objective bcc algorithm after discretization, Ph.D. dissertation, 2011.

[21] L. D. Yang and X. Lu, "Dynamic repair recovery strategy of multiple faults for active distribution network based on multiagent system," Proceedings of the CSEE, vol. 37, no. 23, pp. 6855-6865, 2017.

[22] Q. H. Chen and C. Li, "Evaluation of typhoon resilience of distribution network considering grid reconstruction and disaster recovery," Automation of Electric Power Systems, vol. 42, no. 6, pp. 47-52, 2018.

[23] M. Madhiarasan, "Accurate prediction of different forecast horizons wind speed using a recursive radial basis function neural network," Protection and Control of Modern Power Systems, vol. 5, no. 1, pp. 1-9, 2020.

[24] P. Javanbakht and S. Mohagheghi, "A risk-averse securityconstrained optimal power flow for a power grid subject to hurricanes," Electric Power Systems Research, vol. 116, pp. 408-418, 2014.

[25] S. M. Quiring, L. Zhu, and S. D. Guikema, "Importance of soil and elevation characteristics for modeling hurricane-induced power outages," Natural Hazards, vol. 58, no. 1, pp. 365-390, 2011.

[26] A. M. Salman, Y. Li, and M. G. Stewart, "Evaluating system reliability and targeted hardening strategies of power distribution systems subjected to hurricanes," Reliability Engineering \& System Safety, vol. 144, pp. 319-333, 2015. 
[27] L. Aihua, Research on the dynamics evolution model of urban disaster chain and the risk assessment method of disaster chain, Ph.D. dissertation, Central South University, Changsha, China, 2013.

[28] F. Schulz, D. Wagner, and K. Weihe, "Dijkstra's algorithm online: an empirical case study from public railroad transport," Journal of Experimental Algorithmics (JEA), vol. 5, no. 12, 2000.

[29] S. fangfang, Typhoon damage estimation and prediction of wind vulnerable structures, Ph.D. dissertation, Harbin Institute of Technology, Harbin, China, 2010.

[30] X. Chen, W. Wu, B. Zhang, and X. Shi, "A robust approach for active distribution network restoration based on scenario techniques considering load and dg uncertainties," in Proceedings of the 2016 IEEE Power and Energy Society General Meeting (PESGM), pp. 1-5, IEEE, Boston, MA, USA, July 2016.

[31] M. Zhou, "Optimal algorithm for system reconstruction (utility theory-based rush repair strategy of post-disaster distribution networks with the real-time adjustment)," Proceedings of the CSEE, vol. 23, no. 4, pp. 67-70, 2003.

[32] Z. Jing, "Utility theory-based rush repair strategy of postdisaster distribution networks with the real-time adjustment," Master's thesis, Yanshan University, Qinhuangdao, China, 2014.

[33] R. Francis and B. Bekera, "A metric and frameworks for resilience analysis of engineered and infrastructure systems," Reliability Engineering \& System Safety, vol. 121, pp. 90-103, 2014.

[34] X. Zhiwen, "Emergency resources from the perspective of resilience," Master's thesis, University of Chinese Academy of Sciences, Beijing, China, 2018.

[35] X. Zhou, S. Ge, T. Li et al., "Research on analysis methods and improvement measures of distribution network resilience under extreme weather conditions," Proceedings of the CSEE, vol. 38, no. 2, pp. 505-513, 2018.

[36] C. Nan and G. Sansavini, "A quantitative method for assessing resilience of interdependent infrastructures," Reliability Engineering \& System Safety, vol. 157, pp. 35-53, 2017.

[37] L. Cong, "Studies on optimal control of power system restoration after blackout," Master's thesis, Beijing Jiaotong University, Beijing, China, 2016.

[38] W. Y. Lu, "Reconfiguration of distribution network after fault based on improved ant colony algorithm," in Proceedings of the 24th Annual Conference of Power System and Automation in Chinese Universities, Beijing, China, October 2008.

[39] L. Weijia, Decision-making and optimization strategies for power system restoration, Ph.D. dissertation, Zhejiang University, Zhejiang, China, 2016. 